\title{
Theory of $\mathrm{pp} / \mathrm{pA} / \mathrm{small}$ systems
}

\author{
Piotr Bożek* \\ AGH University of Science and Technology, Faculty of Physics and Applied Computer Science, \\ al. Mickiewicza 30, 30-059 Krakow, Poland \\ E-mail: piotr.bozek@fis.agh.edu.pl
}

\section{Wojciech Broniowski}

The H. Niewodniczański Institute of Nuclear Physics, Polish Academy of Sciences, 31-342

Krakow, Poland

Institute of Physics, Jan Kochanowski University, 25-406 Kielce, Poland

E-mail: wojciech.broniowski@ifj.edu.pl

Experimental results on particle correlations in small collisions system show signs of collectivity. These observations can be understood as a result of final state interaction. The predictions of the relativistic viscous hydrodynamic model compare well to experiment for several observables involving particle with soft momenta.

Fourth Annual Large Hadron Collider Physics

13-18 June 2016

Lund, Sweden

${ }^{*}$ Speaker. 


\section{Introduction}

The formation of strongly interacting matter in relativistic heavy-ion collisions is evidenced by experiments at the BNL Relativistic Heavy Ion Collider (RHIC) and at the CERN Large Hadron Collider (LHC) $[1,2,3,4,5,6,7,8]$. The existence of a fireball of dense matter enables the development of collective expansion, a final state interaction effect. Collisions with small projectiles, $\mathrm{p}+\mathrm{p}, \mathrm{p}+\mathrm{A}$, and $\mathrm{d}+\mathrm{A}$ were expected to show a different behavior, with negligible final state interactions. In the field of heavy-ion collisions small collisions systems were studied as reference systems and to assess the so called cold nuclear matter effects.

In high multiplicity $\mathrm{p}+\mathrm{p}$ and $\mathrm{p}+\mathrm{Pb}$ events at the LHC structures in the two-particle correlation function have been observed similar as in heavy-ion collisions $[9,10]$. The two dimensional correlation function in relative pseudorapidity $\Delta \eta$ and relative azimuthal angle $\Delta \phi$ shows a ridge for $\Delta \phi \simeq 0$, elongated along $\Delta \eta$. This ridge structure has been interpreted as due to the initial state correlation in small collision systems $[11,12,13,14,15,16]$. In this paper we follow the interpretation of the ridge-like structures as due to hydrodynamic expansion in the final state $[17,18,19]$. The azimuthal asymmetry of particle spectra in $\mathrm{p}+\mathrm{Pb}$ has been quantitatively predicted before the experiment in the viscous hydrodynamic model [20].

\section{Collective flow in small collision systems}

In this next section we review basic observations for soft momenta particles emitted in small collision systems and discuss the appearance of effects due to hydrodynamic expansion of the fireball.

\subsection{Elliptic and triangular flow}
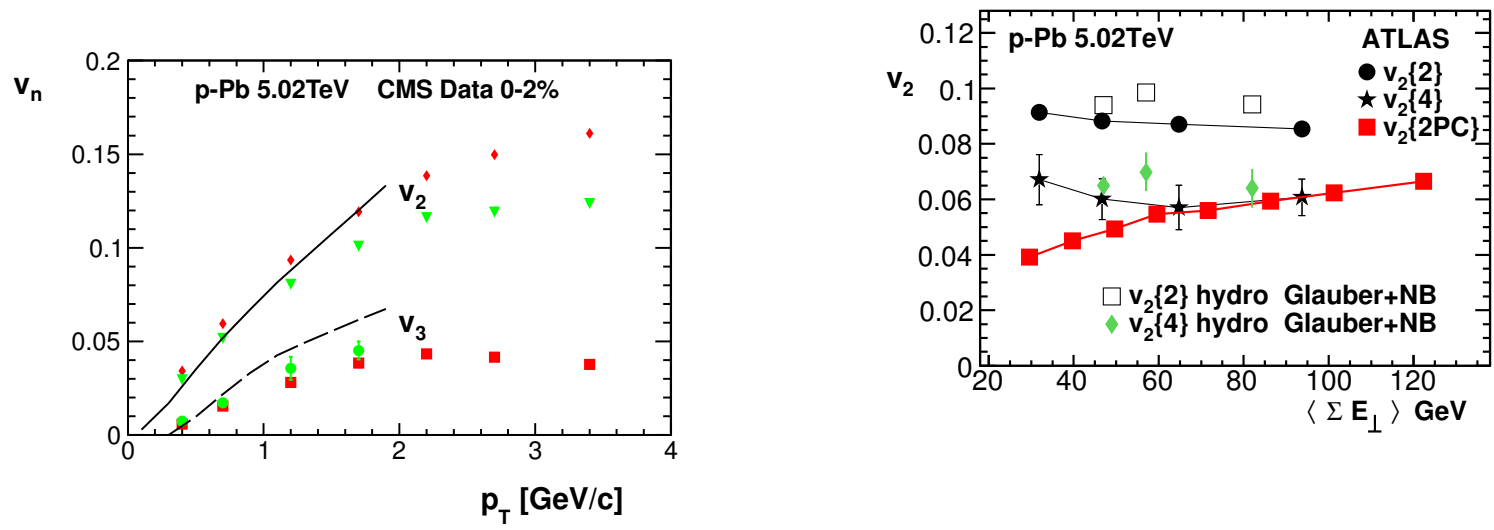

Figure 1: (left panel) Elliptic and triangular flow of charged particles, data from the CMS Collaboration compared to hydrodynamic model results (from [21]). (right panel) Elliptic flow of charged particles measured by the ATLAS Collaboration [22] using three different methods, also are shown the predictions of the hydrodynamic model for $v_{2}$ using the second and fourth cumulant (from [23]). 
A number of independent calculations within the hydrodynamic model in $\mathrm{p}+\mathrm{Pb}$ collisions predict a sizable elliptic $v_{2}$ and triangular $v_{3}$ flow coefficients [20, 23, 24, 25, 26, 27, 28, 29, 30, 31]. In $\mathrm{p}+\mathrm{Pb}$ collisions initial azimuthal deformations of the fireball are generated by fluctuations. The magnitude of fluctuations in the Glauber Monte Carlo model and in the EPOS model yield a flow asymmetry compatible with the data (Fig. 1). The centrality dependence of the elliptic flow coefficient (Fig. 1, right panel) is qualitatively well reproduced in the model. The flat centrality dependence results from a cancellation of the decreasing initial elliptic deformation and an increasing hydrodynamic response with multiplicity.

\subsection{Hierarchy of $v_{2}$ and $v_{3}$ in $\mathbf{p}+\mathbf{A}, \mathbf{d}+\mathbf{A}, \mathbf{H e}+\mathbf{A}$}

The initial geometry in $\mathrm{d}+\mathrm{A}$ collisions is determined mainly by the deformation of the nucleon projectile and fluctuations (Fig. 2, left panel). The initial geometry is better controlled than in p+A collisions and can be tuned using multiplicity cuts [20]. The idea of using small deformed projectiles has been used in experimental studies at RHIC energies [32]. The data is well described by hydrodynamic models [26, 33] (Fig. 2, right panel).
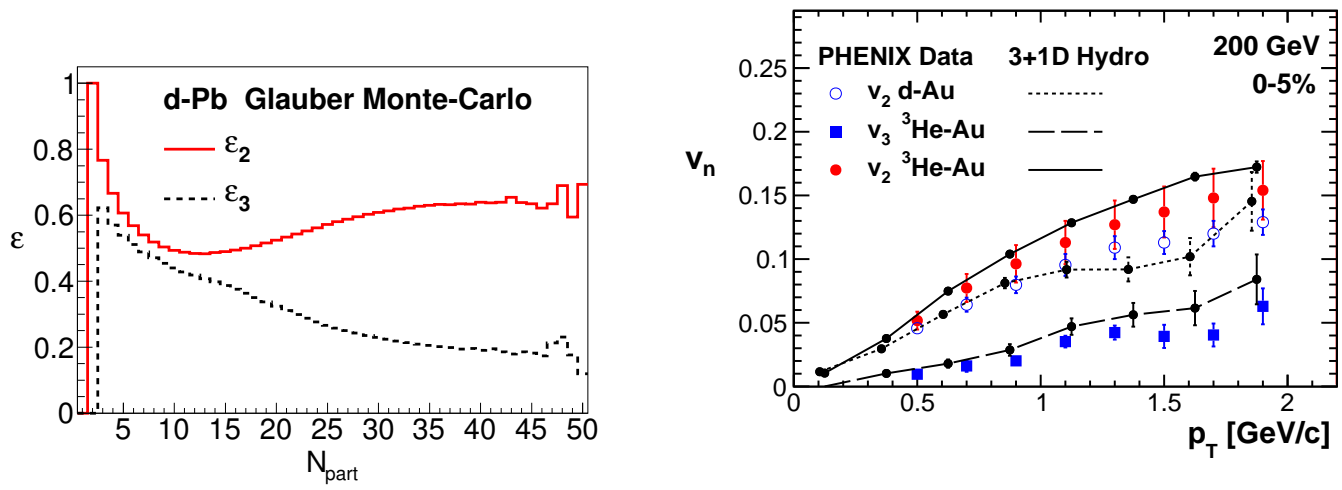

Figure 2: (left panel) Large elliptic deformation in d-Pb collisions, predicted in the Glauber Monte Carlo model (from [20]). Elliptic and triangular flow in $\mathrm{He}+\mathrm{Au}$ and $\mathrm{d}+\mathrm{Au}$ collisions, hydrodynamic model compared to PHENIX Collaboration data [32] (from [33]).

\subsection{Flow from higher cumulants}

In small system collisions the multiplicity is smaller than in typical heavy-ion collisions. This causes difficulties in separating the flow and non-flow contributions to particle correlations. Measurements with reduced non-flow contribution can be performed using higher order cumulants $[34,35]$. The flow coefficients from higher order cumulants can be related to higher moments of the initial eccentricity. In $\mathrm{p}+\mathrm{Pb}$ collisions all higher cumulants are expected to be of similar magnitude, only the second moment is larger. Such a hierarchy has been observed experimentally [22, 36, 37, 38], with $v_{2}\{2\}>v_{2}\{4\} \simeq v_{2}\{6\} \simeq v_{2}\{8\}$ (Fig. 1, right panel). Additional relations between higher order cumulants can be derived in models of the initial state [39]. These relations are consistent with experiment within errors. 


\subsection{Interferometry radii}

The effective size of the emission source can be measured using interferometry correlations. In the hydrodynamic model the effective size decreases with increasing pion pair momentum. A pion pair with large momentum is emitted from a smaller fragment of the fluid with a large collective velocity.
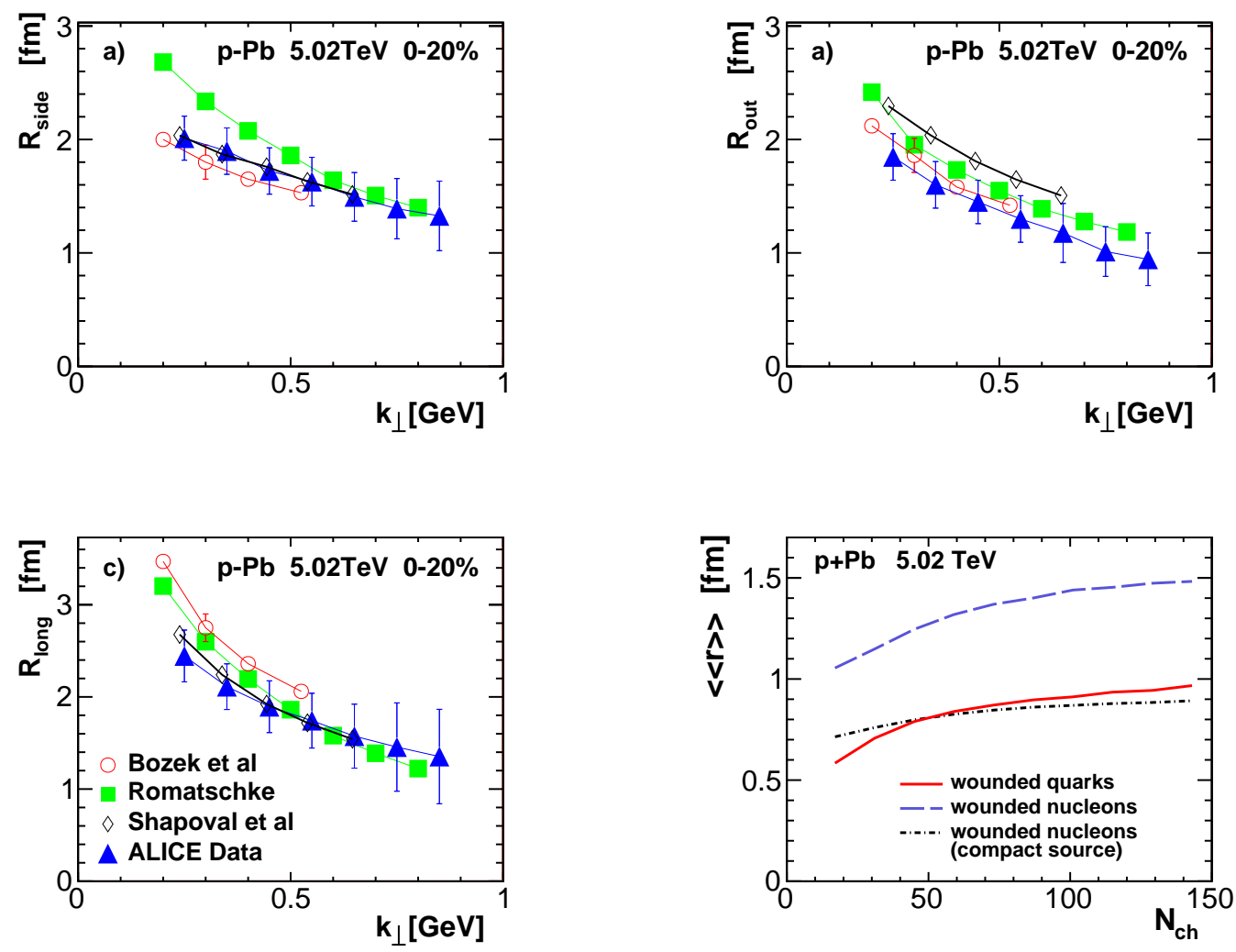

Figure 3: Interferometry radii $R_{\text {side }}$ (upper-left panel), $R_{\text {out }}$ (upper-right panel) and $R_{\text {long }}$ (lower-left panel) as a function of the pair transverse momentum for $\mathrm{p}+\mathrm{Pb}$ collisions calculated in the hydrodynamic models [40, 41, 29], data from the ALICE Collaboration [42]. (lower-right panel) The r.m.s. radius of the source in $\mathrm{p}+\mathrm{Pb}$ collisions from the Wounded nucleon and the wounded quark Glauber model (from [43]).

Model calculations reproduce the experimental interferometry radii in $\mathrm{p}+\mathrm{Pb}$ and $\mathrm{d}+\mathrm{Au}$ collisions fairly well [40, 44, 41, 29] (Fig. 3). The agreement is best when a small initial size of the fireball in $\mathrm{p}+\mathrm{Pb}$ collisions is chosen. The small initial size is naturally explained in the wounded quark Glauber model [43] (Fig. 3, lower-right panel).

\subsection{Factorization of azimuthal correlations}

A collective flow paradigm requires an approximate factorization of two-particle azimuthal correlations in transverse momentum and rapidity [45, 46]. A small breaking of factorization in hydrodynamic models is possible due to fluctuations in the initial state. Experiments confirm that 
factorization is approximately fulfilled for $p_{\perp}<3 \mathrm{GeV}$ [47], and the agreement with model calculations is satisfactory [28].

\subsection{Mass splitting of $v_{2}$}

In hydrodynamics the effect of the collective flow depends on the mass of the particle. The momentum dependent flow coefficients $v_{n}\left(p_{\perp}\right)$ are different for particles of different masses (pions, kaons, protons).
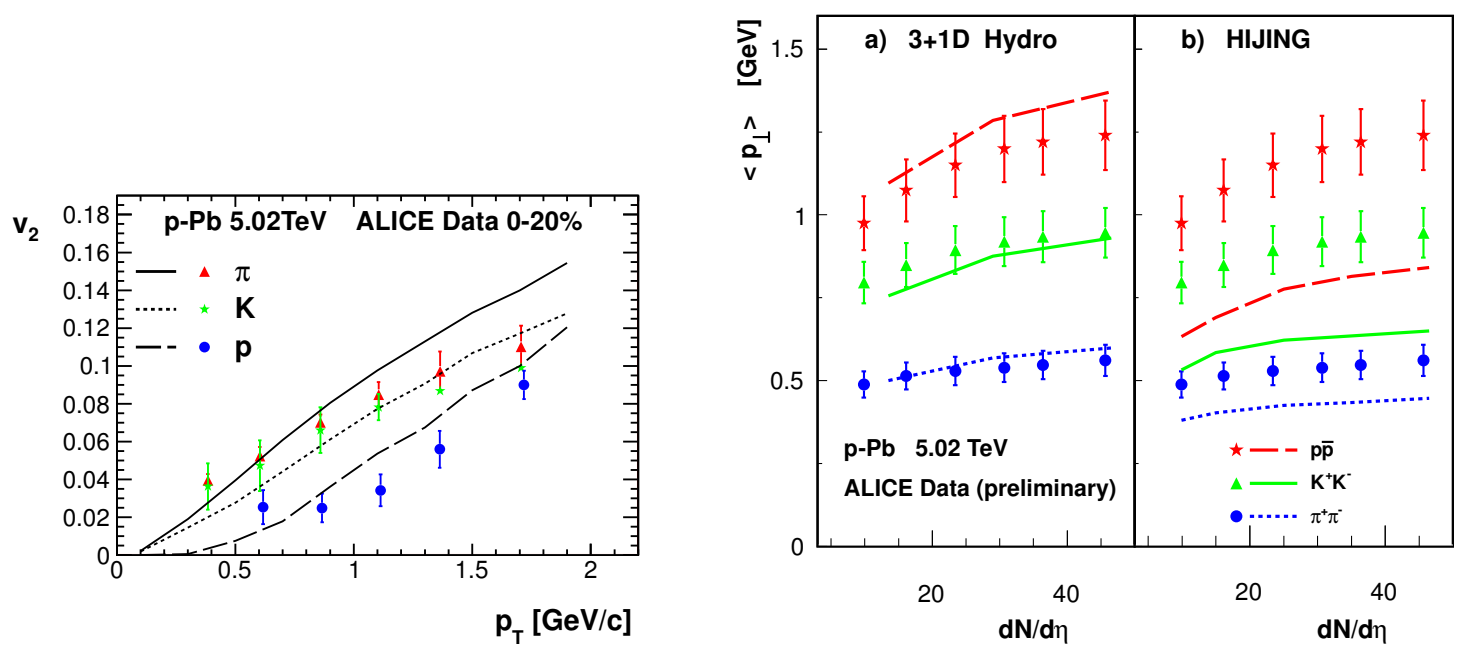

Figure 4: (left panel) Elliptic flow of identified particles in $\mathrm{p}+\mathrm{Pb}$ collisions for the hydrodynamic model compared to ALICE Collaboration data [48]. (right panel) Average transverse momentum of identified particles in $\mathrm{p}+\mathrm{Pb}$ collisions form the HIJING model, from the hydrodynamic model and data from the ALICE Collaboration [49] (from [21]).

The mass splitting of the elliptic flow coefficient is observed experimentally in $\mathrm{p}+\mathrm{Pb}$ [48] and $\mathrm{d}+\mathrm{Au}$ collisions [50]. The effect is reproduced by simulations in the hydrodynamic model [21, 26, 27] (Fig. 4, left panel).

\subsection{Multiplicity dependence and mass hierarchy of spectra}

The velocity of a particle emitted from a fluid element is composed of two contributions, the collective velocity of the fluid and the thermal motion of the particle in the fluid rest frame. Consequently particle of higher mass get a stronger push from the collective flow. A characteristic shift in particle spectra is observed with increasing particle mass [51, 49]. The same effect leads to a mass hierarchy of the average transverse momentum in $\mathrm{p}+\mathrm{Pb}$ collisions (Fig. 4, right panel).

\section{Hydrodynamics in small systems}

Experimental observations in relativistic collisions with small systems indicate that collective expansion occurs in the final state of the collision. The results can be quantitatively understood using relativistic hydrodynamics. This raises the question about the applicability of hydrodynamics 
in systems with large gradient of density $[30,52]$. The most important deviations from equilibrium are visible in the first stage of the expansion, where large asymmetry between transverse and longitudinal pressure appears $[53,54]$.

In strong and weak interaction models of the early dynamics $[55,56]$ hydrodynamics starts to be a good approximation already in phase with large pressure asymmetry. Moreover, the pressure asymmetry has a minor effect on the transverse flow generated in the expansion $[57,58]$.

The breakdown of hydrodynamics at freeze-out makes the model questionable if the mean free path becomes as large as the system size. It has been noticed that cascade models can produce a flow asymmetry without hydrodynamic expansion [59, 60]. On the other hand, it should be noted that numerical simulations in strongly interacting theories show that hydrodynamics applies in systems as small a $R \simeq 1 / T$ [61].

The status of possible collective flow in $\mathrm{p}+\mathrm{p}$ interactions is much less certain than in $\mathrm{p}+\mathrm{Pb}$ collisions. In high multiplicity $\mathrm{p}+\mathrm{p}$ collisions some effects of collective behavior are visible [ 9 , 62, 63]. Several applications of the hydrodynamic model for $\mathrm{p}+\mathrm{p}$ collisions are published[64, 65, $66,67,18,68]$. While the magnitude and the centrality dependence of the flow coefficients is as expected in models assuming fluctuations of the fireball shape in $p+p$ collisions [69, 70], detailed predictions require further understanding of the subnuclear structure of the initial state [71].

\section{Acknowledgments}

Research supported by the Polish Ministry of Science and Higher Education, (MNiSW), by the National Science Centre, Poland grants DEC-2015/17/B/ST2/00101 and DEC-2012/06/A/ST2/00390 and

\section{References}

[1] I. Arsene et al. (BRAHMS), Nucl. Phys. A757, 1 (2005)

[2] B. B. Back et al. (PHOBOS), Nucl. Phys. A757, 28 (2005)

[3] J. Adams et al. (STAR Collaboration), Nucl. Phys. A757, 102 (2005)

[4] K. Adcox et al. (PHENIX Collaboration), Nucl. Phys. A757, 184 (2005)

[5] K. Aamodt et al. (ALICE Collaboration), Phys. Rev. Lett. 105, 252302 (2010)

[6] K. Aamodt et al. (ALICE Collaboration), Phys. Lett. B696, 328 (2011)

[7] G. Aad et al. (ATLAS Collaboration), Phys. Rev. Lett. 105, 252303 (2010)

[8] S. Chatrchyan et al. (CMS), Phys. Rev. C84, 024906 (2011)

[9] V. Khachatryan et al. (CMS), JHEP 09, 091 (2010)

[10] S. Chatrchyan et al. (CMS Collaboration), Phys. Lett. B718, 795 (2013)

[11] K. Dusling and R. Venugopalan, Phys. Rev. Lett. 108, 262001 (2012)

[12] K. Dusling and R. Venugopalan, Phys. Rev. D 87, 054014 (2013)

[13] K. Dusling and R. Venugopalan, Phys. Rev. D 87, 051502 (2013)

[14] Y. V. Kovchegov and D. E. Wertepny, Nucl.Phys. A906, 50 (2013) 
[15] A. Kovner and M. Lublinsky, Int. J. Mod. Phys. E Vol. 22 (2013)

[16] K. Dusling and R. Venugopalan, Phys. Rev. D87, 094034 (2013)

[17] P. Bożek, Eur.Phys.J. C71, 1530 (2011)

[18] K. Werner, I. Karpenko, and T. Pierog, Phys. Rev. Lett. 106, 122004 (2011)

[19] P. Bożek and W. Broniowski, Phys. Lett. B718, 1557 (2013)

[20] P. Bożek, Phys. Rev. C85, 014911 (2012)

[21] P. Bożek, W. Broniowski, and G. Torrieri, Phys. Rev. Lett. 111, 172303 (2013)

[22] G. Aad et al. (ATLAS Collaboration), Phys. Lett. B725, 60 (2013)

[23] P. Bożek and W. Broniowski, Phys. Rev. C88, 014903 (2013)

[24] A. Bzdak, B. Schenke, P. Tribedy, and R. Venugopalan, Phys. Rev. C87, 064906 (2013)

[25] G.-Y. Qin and B. Müller, Phys. Rev. C89, 044902 (2014)

[26] J. Nagle, A. Adare, S. Beckman, T. Koblesky, J. O. Koop, et al., Phys.Rev.Lett. 113, 112301 (2014)

[27] K. Werner, M. Bleicher, B. Guiot, I. Karpenko, and T. Pierog, Phys. Rev. Lett. 112, 232301 (2014)

[28] I. Kozlov, M. Luzum, G. Denicol, S. Jeon, and C. Gale(2014), arXiv:1405.3976 [nucl-th]

[29] P. Romatschke, Eur. Phys. J. C75, 305 (2015)

[30] H. Niemi and G. S. Denicol(2014), arXiv:1404.7327 [nucl-th]

[31] C. Shen, J. F. Paquet, G. S. Denicol, S. Jeon, and C. Gale, Phys. Rev. Lett. 116, 072301 (2016)

[32] A. Adare et al. (PHENIX)(2015), arXiv:1507.06273 [nucl-ex]

[33] P. Bozek and W. Broniowski, Phys. Lett. B747, 135 (2015)

[34] N. Borghini, P. M. Dinh, and J.-Y. Ollitrault, Phys. Rev. C63, 054906 (2001)

[35] N. Borghini, P. M. Dinh, and J.-Y. Ollitrault, Phys.Rev. C64, 054901 (2001)

[36] S. Chatrchyan et al. (CMS Collaboration), Phys. Lett. B724, 213 (2013)

[37] V. Khachatryan et al. (CMS), Phys. Rev. Lett. 115, 012301 (2015)

[38] B. B. Abelev et al. (ALICE), Phys. Rev. C90, 054901 (2014)

[39] L. Yan and J.-Y. Ollitrault, Phys. Rev. Lett. 112, 082301 (2014)

[40] P. Bożek and W. Broniowski, Phys. Lett. B720, 250 (2013)

[41] P. Bożek, Phys.Rev. C90, 064913 (2014)

[42] J. Adam et al. (ALICE), Phys. Rev. C91, 034906 (2015)

[43] P. Bożek, W. Broniowski, and M. Rybczyński, Phys. Rev. C94, 014902 (2016)

[44] V. M. Shapoval, P. Braun-Munzinger, I. A. Karpenko, and Yu. M. Sinyukov, Phys. Lett. B725, 139 (2013)

[45] F. G. Gardim, F. Grassi, M. Luzum, and J.-Y. Ollitrault, Phys.Rev. C87, 031901 (2013)

[46] P. Bożek, W. Broniowski, and J. Moreira, Phys. Rev. C83, 034911 (2011)

[47] V. Khachatryan et al. (CMS Collaboration), Phys. Rev. C92, 034911 (2015) 
[48] B. B. Abelev et al. (ALICE Collaboration), Phys. Lett. B726, 164 (2013)

[49] B. B. Abelev et al. (ALICE Collaboration), Phys.Lett. B728, 25 (2014)

[50] N. Ajitanand et al. (PHENIX), Nucl.Phys. A931, 1082 (2014)

[51] S. Chatrchyan et al. (CMS), Eur. Phys. J. C74, 2847 (2014)

[52] M. Nopoush, M. Strickland, R. Ryblewski, D. Bazow, U. Heinz, and M. Martinez, Phys. Rev. C92, $044912(2015)$

[53] W. Florkowski and R. Ryblewski, Phys. Rev. C83, 034907 (2011)

[54] M. Martinez and M. Strickland, Nucl. Phys. A848, 183 (2010)

[55] M. P. Heller, R. A. Janik, and P. Witaszczyk, Phys. Rev. Lett. 108, 201602 (2012)

[56] L. Keegan, A. Kurkela, A. Mazeliauskas, and D. Teaney, JHEP 08, 171 (2016)

[57] J. Vredevoogd and S. Pratt, Phys. Rev. C79, 044915 (2009)

[58] P. Bożek and I. Wyskiel-Piekarska, Phys. Rev. C83, 024910 (2011)

[59] A. Bzdak and G.-L. Ma, Phys.Rev.Lett. 113, 252301 (2014)

[60] L. He, T. Edmonds, Z.-W. Lin, F. Liu, D. Molnar, and F. Wang, Phys. Lett. B753, 506 (2016)

[61] P. M. Chesler, Phys. Rev. Lett. 115, 241602 (2015)

[62] V. Khachatryan et al. (CMS), Phys. Rev. Lett. 116, 172302 (2016)

[63] G. Aad et al. (ATLAS), Phys. Rev. Lett. 116, 172301 (2016)

[64] M. Luzum and P. Romatschke, Phys. Rev. Lett. 103, 262302 (2009)

[65] A. K. Chaudhuri, Phys. Lett. B692, 15 (2010)

[66] P. Bożek, Acta Phys. Pol. B41, 837 (2010)

[67] W.-T. Deng, Z. Xu, and C. Greiner, Phys. Lett. B711, 301 (2012)

[68] T. Kalaydzhyan and E. Shuryak, Phys. Rev. C91, 054913 (2015)

[69] J. Casalderrey-Solana and U. A. Wiedemann, Phys. Rev. Lett. 104, 102301 (2010)

[70] E. Avsar, C. Flensburg, Y. Hatta, J.-Y. Ollitrault, and T. Ueda, Phys. Lett. B702, 394 (2011)

[71] B. Schenke and R. Venugopalan, Phys. Rev. Lett. 113, 102301 (2014) 